\title{
Repeatability of cough-related variables during fog challenges at threshold and suprathreshold stimulus intensity in humans
}

\author{
G.A. Fontana*, T. Pantaleo ${ }^{+}$, F. Lavorini*, N.M. Maluccio*, D. Mutolo ${ }^{+}$, M. Pistolesi*
}

\begin{abstract}
Repeatability of cough-related variables during fog challenges at threshold and suprathreshold stimulus intensity in humans. G.A. Fontana, T. Pantaleo, F. Lavorini, N.M. Maluccio, D. Mutolo, M. Pistolesi. (C)ERS Journals Ltd 1999.

ABSTRACT: Cough-related variables such as cough frequency, time to onset (i.e. the time until the first cough occurs) and the cough index (i.e. the ratio between the cough frequency and the time to onset) may be important when interpreting results of cough challenges for therapeutic interventions or for comparative research purposes. Nevertheless, repeatability (or reproducibility) for these widely used variables has been poorly studied.

In thirty normal subjects, coughing was induced by inhalation of threshold $(T)$ and suprathreshold $(1.6 \times T)$ concentrations of ultrasonically nebulized distilled water (fog). Cough threshold was taken as the lowest fog concentration that evoked at least one cough effort during two challenges separated by a 30-min interval. During challenges performed at both threshold and suprathreshold stimulus intensity, cough frequency, time to onset, and the cough index were assessed; within-subject repeatability for these variables was subsequently evaluated.

Median \pm interquartile range cough threshold value was $0.9 \pm 0.5 \mathrm{~mL} \cdot \mathrm{min}^{-1}$. During the two challenges performed to assess cough threshold, the mean \pm SD values of cough frequency, time to onset, and cough index were similar $(5.0 \pm 2.7$ and $5.3 \pm 3.1$ coughs. $\min ^{-1}, 32.4 \pm 13.3$ and $32.9 \pm 13.6 \mathrm{~s}$, and $0.2 \pm 0.2$ and $0.2 \pm 0.2$, respectively). However, none of these cough-related variables proved to be sufficiently repeatable. During the two challenges performed at suprathreshold stimulus intensity, mean values of cough frequency, time to onset, and cough index were also similar $(20.0 \pm 9.0$ and $18.2 \pm 10.2$ coughs min $^{-1}, 13.5 \pm 5.8$ and $12.0 \pm 4.62 \mathrm{~s}$, and $1.7 \pm 1.0$ and $1.8 \pm 1.2$ ); furthermore, all considered variable of suprathreshold challenge turned out to be reproducible.

In conclusion, during fog challenges at threshold stimulus intensity, cough frequency, time to onset and cough index cannot reliably be used for evaluating cough responses. However, these cough-related variables may represent useful and reliable research tools in the evaluation of suprathreshold cough responses.

Eur Respir J 1999; 13: 1447-1450.
\end{abstract}

*Dipartimento di Fisiopatologia Clinica, Sezione di Fisiopatologia Respiratoria, and ${ }^{+}$Dipartimento di Scienze Fisiologiche, Università di Firenze, Florence, Italy.

Correspondence: G.A. Fontana

Dipartimento di Fisiopatologia Clinica

Sezione di Fisiopatologia Respiratoria

Viale G.B. Morgagni, 85

50134 Firenze

Italy

Fax: 390554223202

Keywords: Airway reflexes

cough

ultrasonically nebulized distilled water

Received: October 121998

Accepted after revision February 191999

The objective assessment of cough may be important to investigate the neural mechanisms triggering cough as well as those underlying the cough response; it may also be of interest for studies on antitussive agents. Cough can be induced by inhalation of pharmacological and nonpharmacological agents, both in patients and in healthy subjects [1-9]. The former agents include citric acid, capsaicin, histamine, bradykinin, and prostaglandins [1$3]$, and the latter basically refer to ultrasonically nebulized water (fog) and solutions low in permeant anion content $[1,4-9]$. Cough receptor sensitivity has often been evaluated in terms of the lowest concentration of a tussigenic agent capable of evoking a predetermined number of expiratory thrusts $[2,7,9]$. More recently, cough threshold has been measured as the lowest fog concentration capable of eliciting at least one cough effort during two fog challenges separated by a 30 -min interval $[4,5]$. Short- and long-term repeatability, or reproducibility, of cough threshold has also been demonstrated [4]. Additional cough-related variables, such as cough frequency, the time which elapses between the beginning of inha- lation of the tussigenic agent and the appearance of the first cough effort (time to onset), as well as the cough index (i.e. the ratio between cough frequency and the time to onset) have also been adopted in different experimental conditions to get insights into the sensitivity of cough receptors or to quantify cough responses [2, 10]. Alhough the use of these variables has provided valuable information on mechanisms implicated in cough sensitivity $[2,7,9,10]$, their within-patient repeatability has scarcely been considered. This study was carried out to evaluate the short-term repeatability of cough-related variables during coughing induced by inhalation of threshold and suprathreshold fog concentrations in healthy subjects.

\section{Material and methods}

\section{Subjects}

Thirty healthy nonsmoking volunteers ( 24 males and six females, mean age 38 yrs, range 25-68 yrs) who coughed 
in response to preliminary fog challenges took part in the study. Subjects had already participated in previous cough studies performed in the authors' laboratory [4], and were therefore fully aware of the procedure to be employed, but not of the purposes of the present study. The protocol was approved by the local ethical committee; individual informed consent was obtained from each participant.

\section{Experimental procedures and protocol}

Experiments were carried out according to procedures previously described [4, 5]. Cough was induced by inhalation of fog produced by a Mist- $\mathrm{O}_{2}$-Gen EN143A ultrasonic nebulizer (Mist- $\mathrm{O}_{2}$-Gen Equipment Company, Oakland, CA, USA) whose output could be adjusted in steps corresponding to $5 \%$ of the maximum attainable direct current (DC) output. The range of nebulizer outputs employed in the present study was $0.08-4.45 \mathrm{~mL} \cdot \mathrm{min}^{-1}$ [4]. Cough occurrence was monitored by means of the electromyographic (EMG) activity of the abdominal muscles recorded via surface $\mathrm{Ag}-\mathrm{AgCl}$ electrodes from the anterolateral region of the abdomen $[4,5]$. The EMG signals were processed and recorded as described elsewhere $[4,5]$ but not quantitatively analysed. Participants were comfortably seated on a dentist's chair and were repeatedly reminded to relax and breathe normally with as constant a pattern as possible. After a few minutes of rest, they tidally inhaled for $1 \mathrm{~min}$ the fog concentration produced at the scheduled nebulizer output. Cough threshold was taken as the lowest fog concentration capable of evoking at least one cough effort during two distinct fog challenges separated by a 30 -min interval $[4,5]$. One hour after cough threshold assessment, participants were requested to inhale, during two additional challenges separated by a 30-min interval and performed with the same procedure as that used for assessing cough threshold, the fog concentration corresponding to 1.6-times the previously determined threshold concentration.

\section{Data analysis}

During all challenges at threshold and suprathreshold levels, cough frequency and time to onset were measured; cough index was subsequently calculated. The repeatability (or reproducibility) of all considered variables was evaluated by using the method of BLAND and AltMan [11]. With this method, repeatability can be accepted when no more than $5 \%$ of the calculated differences between values of each variable obtained during the two successive fog challenges exceeded the coefficient of repeatability, which is taken as twice the standard deviation of the differences between pairs of repeated observations. This method can confidently be used only when calculated differences in each variable do not significantly differ (paired Student's t-tests) from zero [11]. Reported values are mean \pm SD unless otherwise stated; in all instances, $\mathrm{p}<0.05$ was taken as significant.

\section{Results}

In the whole population studied, cough threshold values ranged $0.4-1.6$ (median 0.9 ) $\mathrm{mL} \cdot \mathrm{min}^{-1}$; the corresponding lst and $3 \mathrm{rd}$ quartile were 0.8 and $1.3 \mathrm{~mL} \cdot \mathrm{min}^{-1}$ with an interquartile range of $0.5 \mathrm{~mL} \cdot \mathrm{min}^{-1}$.
At threshold stimulus intensity (fig. 1a-c), mean values of cough frequency, time to onset, and cough index recorded during the first and second fog challenge were $5.0 \pm 2.7$ and $5.3 \pm 3.1$ coughs $\cdot \mathrm{min}^{-1}, 32.4 \pm 13.3$ and $32.9 \pm$ $13.6 \mathrm{~s}$, and $0.2 \pm 0.2$ and $0.2 \pm 0.2$, respectively. The corresponding mean differences were $-0.3 \pm 3.8$ coughs $\cdot \mathrm{min}^{-1}$, $-0.5 \pm 17.3 \mathrm{~s}$, and $0 \pm 0.3$, respectively, and did not significantly differ from zero. As depicted in figure $1 \mathrm{a}-\mathrm{c}$, none of the considered variables proved to be repeatable; in fact, for cough frequency, time to onset, and cough index the number of differences exceeding the coefficient of repeatability was $4(13.3 \%), 3(10.0 \%)$, and $4(13.3 \%)$, respectively.

At suprathreshold stimulus intensity (fig. 1d-f), mean values of cough frequency, time to onset and cough index obtained during the first and second fog challenge were $20.0 \pm 9.0$ and $18.2 \pm 10.2$ coughs $\cdot \mathrm{min}^{-1}, 13.5 \pm 5.8$ and $12.0 \pm$ $4.62 \mathrm{~s}, 1.7 \pm 1.0$ and $1.8 \pm 1.2$, respectively. The corresponding mean differences in cough frequency $(1.8 \pm 5.5$ coughs. $\left.\mathrm{min}^{-1}\right)$, time to onset $(1.7 \pm 3.9 \mathrm{~s})$, and cough index $(-0.1 \pm$ 0.5 ) calculated between the first and second suprathreshold fog challenge did not significantly differ from zero. As shown in figures $1 \mathrm{~d}-\mathrm{f}$, none of the differences exceeded the coefficient of repeatability.

\section{Discussion}

This study shows that, at threshold stimulus intensity for the cough reflex, repeatability of cough frequency, time to onset and cough index is poor. In contrast, at higher stimulus intensities, all considered cough-related variables seem to be quite reproducible.

So far, attempts at evaluating the repeatability of coughrelated variables during inhalation of water solutions have yielded conflicting results. For instance, TANAKA et al. [12], using the same statistical method employed here, have found no significant difference in the number of cough efforts elicited during two separate fog inhalation challenges, both in patients and in healthy subjects. With the use of a different statistical procedure, VENTRESCA et al. [13] failed to demonstrate, in eight normal subjects, any significant difference in the number of coughs elicited by two 1-min inhalations of four isosmolar solutions with decreasing chloride concentrations, thus suggesting that this cough challenge determines reproducible responses. Relationships between cough frequency and cough stimulus intensity, however, have been denied by EschenBACHER et al. [6]. Noticeably, while in the study by EscHENBACHER et al. [6] stimulus intensity was at threshold level, in the studies by TANAKA et al. [12] and VENTRESCA et al. [13] cough stimulus was of suprathreshold intensity. Since increasing stimulus intensity improves repeatability of the response, as demonstrated by the present results, it seems likely that the contrasting outcomes reported in these studies are a consequence of differences in the intensity of the cough stimulus used. The reasons underlying repeatability of cough frequency at suprathreshold but not threshold cough stimulus intensity are not immediately clear. However, it can reasonably be expected that, at threshold level, when the neural mechanisms subserving cough are just brought into action, there is a high degree of variability in the overall cough motor response. On the other hand, at suprathreshold levels, these neural 
a)

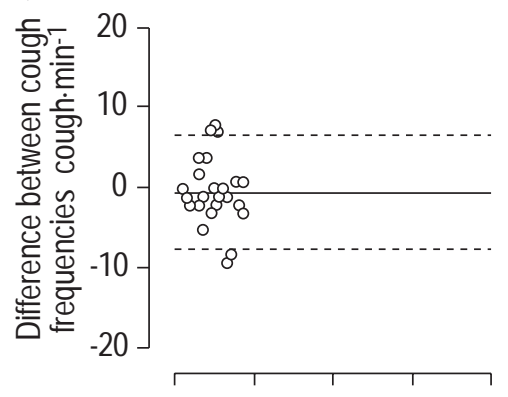

d)

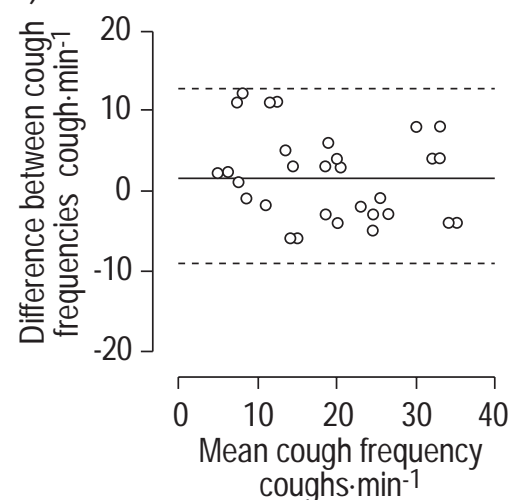

b)

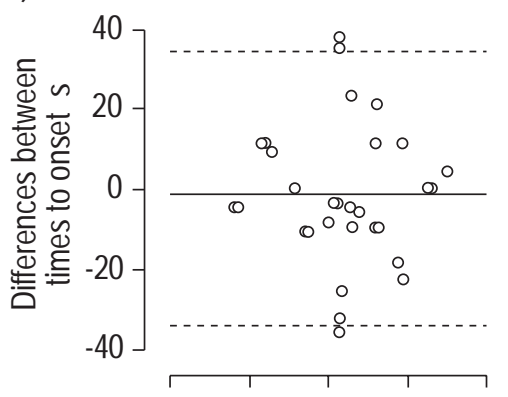

e)

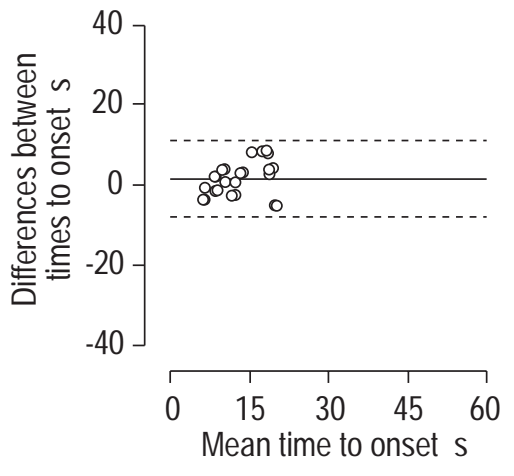

c)

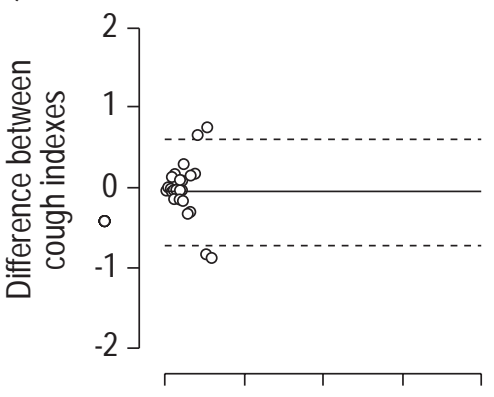

f)

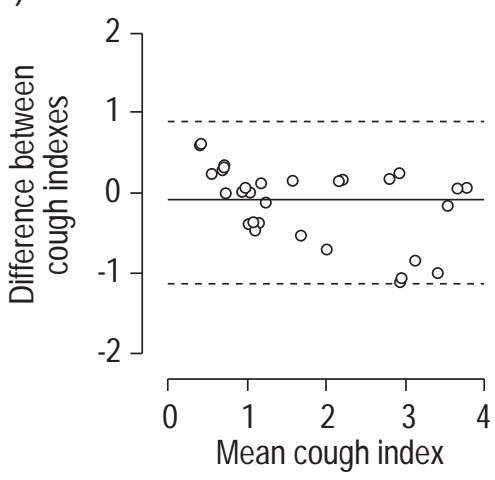

Fig. 1. - Individual differences between values of cough frequency $(a, d)$, time to onset $(b, e)$, and cough index obtained during two consecutive 1-min challenges at threshold $(\mathrm{a}-\mathrm{c})$ and suprathreshold $(\mathrm{d}-\mathrm{f})$ stimulus intensity, plotted against their respective mean values. In a, $\mathrm{c}$, and e, some data points are superimposed due to overlapping values. - : mean difference; - - - : $\pm 2 \mathrm{sD}$.

mechanisms are more extensively or even fully recruited and may provoke more stereotyped and reproducible responses in each subject. The higher number of expiratory thrusts elicited by more intense airway stimulation may also contribute. In fact, with the analytical procedure used for assessing repeatability [11], an increased number of observations may result in a larger scatter of calculated differences (dotted lines of fig. 1) between cough frequency values obtained during the two challenges; a wider variability interval may in turn increase the number of data points comprised in the repeatability range. The fact that the distribution range of mean cough frequency values was found to be wider at suprathreshold stimulus intensity likely reflects between-subject differences in adaptation to the suprathreshold cough stimulus. In fact, adaptation to a suprathreshold cough stimulus may be relatively constant within a given subject, but may display considerable variability among subjects [1]. As regards the time to onset, both the repeatability and the narrower range of value distribution displayed by this variable following suprathreshold cough stimuli are most likely related to the higher amount of stimulant reaching airway cough receptors in a given time interval, thus leading to a faster onset of the cough response. Both at threshold and suprathreshold stimulus intensity, reproducibility and value distribution of the cough index is obviously dependent on its determinants, since the cough index is the ratio between cough frequency and time to onset.

It can be questioned whether or not suprathreshold responses were influenced by prior threshold determination. For methodological reasons, the order of these trials was mandatory. Nevertheless, tachyphylaxis to the cough stimulus, i.e., to the authors' knowledge, the major factor which may influence the outcomes of repeated fog challenges [1], can easily be avoided by allowing subjects to recover for at least $30 \mathrm{~min}$ after the first challenge $[1,4$, 5]. This is supported by the finding that cough threshold values are, in most cases, the same when measured during both of the challenges, performed with a 30-min interval, required for cough threshold assessment. Suprathreshold challenges performed $1 \mathrm{~h}$ after threshold assessment are even less likely to be influenced by tachyphylaxis.

The number of male participants recruited for the present investigation markedly exceeded that of females, suggesting some selection bias. Subjects were recruited from among medical students, technical staff, and nurses; enrolling of female volunteers was generally difficult. Although in both male and female participants' values the distribution range of all considered cough-related variables appeared to be similar, this aspect deserves to be fully elucidated by analysing a wider group of female subjects.

In both the present and previous studies performed in the authors' laboratory $[4,5]$, administration of the threshold cough stimulus may provoke from just one to a few cough efforts. This could suggest that, at least in some instances, the intensity of the administered stimulus was somewhat higher than the actual cough threshold. This relates to the practical impossibility of obtaining reproducible measures of the amount of nebulized water when the nebulizer output is regulated in steps $<5 \%$ of the maximum attainable output. Even if actual threshold values have been overestimated in some instances, it has been demonstrated that cough threshold to fog inhalation can be measured fairly accurately with a high degree of short- and 
long-term repeatability $[4,5]$. In conclusion, it is believed that cough threshold represents the best method for quantification of a subject's sensitivity to the stimulus adopted, and that it should be regarded as an expression of both the functional characteristics of receptors and the responsiveness of central stations involved in the generation of the cough reflex $[4,5]$. Nevertheless, in the evaluation of cough responses elicited by stimuli of suprathreshold intensity, cough frequency, time to onset and cough index may represent useful tools for cough research. This appears to be particularly true when the use of variables which are easily and inexpensively obtained is recommended, and the aim is to evaluate the magnitude of the overall cough response rather than the strength of single expiratory thrusts.

\section{References}

1. Morice AH, Higgins KS, Yeo WW. Adaptation of cough reflex with different types of stimulation. Eur Respir $J$ 1992; 5: 841-847.

2. Pounsford JC, Birch MJ, Saunders KB. Effects of bronchodilators on the cough response to inhaled citric acid in normal and asthmatic subjects. Thorax 1985; 40: 662-667.

3. Choudry NB, Fuller RW, Pride NB. Sensitivity of the human cough reflex: effect of inflammatory mediators prostaglandin $\mathrm{E}_{2}$, bradykinin, and histamine. Am Rev Respir Dis 1989; 140: 137-141.

4. Fontana GA, Pantaleo T, Lavorini F, Boddi V, Panuccio P. A noninvasive electromyographic study on threshold and intensity of cough in human. Eur Respir J 1997; 10: $983-$ 989.

5. Fontana GA, Pantaleo T, Lavorini F, Benvenuti F, Gangemi S. Defective motor control of coughing in Parkinson's disease. Am J Respir Crit Care Med 1998; 158: 458-464.

6. Eschenbacher WL, Boushey HA, Sheppard D. Alteration in osmolarity of inhaled aerosols cause bronchoconstriction and cough, but absence of a permeant anion causes cough alone. Am Rev Respir Dis 1984; 129: 211-215.

7. Higenbottam $\mathrm{T}$, Jackson $\mathrm{M}$, Woolman $\mathrm{P}$, Lowry $\mathrm{R}$, Wallwork J. The cough response to ultrasonically nebulized distilled water in heart-lung transplantation patients. Am Rev Respir Dis 1989; 140: 58-61.

8. Banner AS. Relationship between cough due to hypotonic aerosol and the ventilatory response to $\mathrm{CO}_{2}$ in normal subjects. Am Rev Respir Dis 1988; 137: 647-650.

9. Lowry R, Wood A, Johnson T, Higenbottam T. Antitussive properties of inhaled bronchodilators on induced cough. Chest 1988; 93: 1186-1189.

10. Pounsford JC, Saunders KB. Diurnal variation and adaptation of the cough response to citric acid in normal subjects. Thorax 1985; 40: 657-661.

11. Bland JM, Altman DG. Statistical methods for assessing agreement between two methods of clinical measurements. Lancet 1986; 8: 307-310.

12. Tanaka S, Hirata K, Kudhara N, Yoshikawa J, Takeda T. Effect of loratidine, an $\mathrm{H}_{1}$ antihistamine, on induced cough in non-asthmatic patients with chronic cough. Thorax 1996; 51: 810-814.

13. Ventresca PG, Nichol GM, Barnes PJ, Chung KF. Inhaled furosemide inhibits cough induced by low chloride content solutions but not by capsaicin. Am Rev Respir Dis 1990; 142: 143-146. 